\title{
Effectiveness of Physiotherapy on Psychosocial Factors in COVID-19 Positive Patients: An Interventional Study
}

\author{
Isha Trivedi ${ }^{1}$, Sweety Shah $^{2}$ \\ ${ }^{1}$ Post-Graduate Student, SBB College of Physiotherapy, VS General Hospital, SVP Hospital, Ahmedabad, India \\ ${ }^{2}$ Ph.D., MPT, SBB College of Physiotherapy, VS General Hospital, SVP Hospital, Ahmedabad, India
}

Corresponding Author: Isha Trivedi

\begin{abstract}
Objectives: Corona virus has impacted mental health a lot along with the physical health. The patients have to stay isolated and all alone at hospital for treatment purposes. So the aim of the study was to find out the effectiveness of physiotherapy on psychosocial factors in covid19 positive patients.

Methods: 26 patients were included. Three components of psychosocial health were assessed. Anxiety was assessed with Corona Anxiety Scale, Stress assessed with Corona Stress Scales and Depression assessed with Patient Health Questionnaire (PHQ-9) pre and post physiotherapy interventions.

Results: Significant improvement was seen in all the three components pre and post physiotherapy intervention.

Conclusion: According to the study it is concluded that physiotherapy plays a positive role in management of psychosocial factors in covid19 patients and it should be included as an integral part of treatment protocol in managing the same.
\end{abstract}

Keywords: COVID-19, stress, anxiety, depression, physiotherapy

\section{INTRODUCTION}

COVID-19 is caused by a novel coronavirus, named severe acute respiratory syndrome coronavirus 2 (SARS-CoV-2) [previously provisionally known as 2019 novel coronavirus (2019-nCoV)]. ${ }^{1}$ While certain infectious diseases have been linked to socioeconomic disadvantage, mental health problems, and lower cognitive function, relationships with COVID-19 are either uncertain or untested. ${ }^{2}$

Although there has been substantial attention to measures to identify people with the coronavirus infection, identifying the mental health care needs of people impacted by this pandemic have been relatively neglected. ${ }^{3}$ As evidenced by the previous SARS and MERS outbreaks, viral infections and the subsequent isolation and quarantine can quickly culminate into sleep disturbances, anxiety and depressive episodes.

People have modified their actions because of fear of being discriminated against, for example by avoiding testing for SARS-CoV-2 (anticipated stigma); patients and their families felt judged by others (perceived stigma); infected or exposed persons were excluded, isolated and discriminated against by their household and/or community members (experienced stigma); and some patients might have felt shame and self-rejection (internalised stigma). ${ }^{4}$

COVID-19 positive patients are isolated and have to stay alone for treatment 
purpose in hospital. Along with the physical health, mental health of those patients is assumed to get disturbed which eventually lowers the immunity.

Physical therapy impacts mental health and evidences are suggestive of that, good physical health is always associated with better psychosocial health.

The need of this study is to find out the role of physiotherapy in management of psychosocial factors in covid19 positive patients with the objectives to assess depression, anxiety and stress in covid19 positive patients pre and post physiotherapy treatment protocol.

\section{MATERIALS AND METHODS}

The interventional study was done at SVP Hospital, Ahmedabad. The study comprised of covid19 positive patients aged from 18 to 80 years taken as subjects from covid19 positive ICUs and wards, those referred for physiotherapy. Both male and female patients were included. The study was approved by Ethical review committee. Random sampling was done.

The exclusion criteria was covid19 negative patients, any medically diagnosed mental illness, any neurological Conditions (previous stroke, focal lesions) and pregnant covid19 positive females.

The materials used were covid anxiety scale, covid stress scales, patient health questionnaire (PHQ-9), pulse oximeter, pen and paper.

Prior to the intervention oral consent was taken from the patients. Sample size was calculated according to power analysis and in total 26 patients was included in the study. Physiotherapy intervention was given two times per day and for 14 days to the included patients.

Outcome measures were (i) Covid Anxiety Scale, (ii) Covid Stress Scales and (iii) Patient Health Questionnaire (PHQ-9) which were assessed pre and post physiotherapy intervention.

Covid Anxiety Scale: A pool of 20 candidate items created based on the psychology of fear and anxiety literature (American Psychiatric Association, 2013; Barlow, 1991; Cosmides \& Tooby, 2000; Ekman, 2003; Ohman, 2000). It includes the cognitive (i.e., repetitive thinking; worry; processing biases; dreaming; planning), behavioral (i.e., dysfunctional activities; avoidance; compulsive behaviors), emotional (i.e., fear; anxiety; anger), and physiological (i.e., sleep disturbances; somatic distress; tonic immobility;) dimensions of coronavirus anxiety. ${ }^{3}$ It is a 5-point scale to reflect the frequency of the symptom, ranging from 0 (not at all) to 4 (nearly every day) over the preceding two weeks. It has reliability of 0.88 and validity of 0.93 .

Covid Stress Scales: The CSS were developed to better understand and assess COVID-19-related distress. ${ }^{5}$ It assess covid-related stress and anxiety symptoms: (1) Danger and contamination fears, (2) fears about economic consequences, (3) xenophobia, (4) compulsive checking and reassurance seeking, and (5) traumatic stress symptoms about COVID-19. It has reliability of 0.80 and reliability of 0.90 .

Patient Health Questionnaire (PHQ-9): The depression module of the Patient Health Questionnaire-9 (PHQ-9) is a widely used depression screening instrument in nonpsychiatric settings. ${ }^{6}$ The PHQ-9 is a self-administered version of the depression portion of the PRIME-MD interview, which uses DSM-IV criteria to assess for mental disorders in primary care. A score of 5-9 is considered minimal depression, 10-14 is considered mild major, 15-19 is moderate major, and $\geq 20$ is severe major. ${ }^{7}$ It has reliability of 0.89 and validity of 0.73 .

Physiotherapy regime included of Deep breathing exercises, Diaphragmatic breathing technique, Thoracic expansion exercises, Segmental expansion exercises, Expiration by Red Rubber Balloon, Positioning, Active limb physiotherapy exercises and Walking in ward and ICU. 
In deep breathing exercises, the patients were instructed to "Breathe in slowly and as deeply as possible through your nose or mouth to take as much air as possible into your lungs, hold your breath for a while, and then breathe the air out slowly through your mouth/device until you have some air left in your lungs". ${ }^{8}$

For diaphragmatic breathing, they were instructed to perform a nasal inspiration moving predominantly the abdomen, reducing the movement of the rib cage. ${ }^{9}$ Diaphragmatic breathing technique is performed in semi-fowler's position.

The thoracic expansion exercises are incorporated into the regimen, as they are believed to assist in the loosening and removal of pulmonary secretions, improve collateral ventilation and also, in conjunction with the relaxed breathing, prevent the occurrence of fatigue, bronchospasm and oxygen desaturation (Pryor and Webber, 1979; Pryor, Webber and Hodson, 1990; Webber and Pryor, 1993). 10 Inspiration was instructed to perform with upper limb elevation and expiration was instructed to perform with upper limb depression/or back to starting position.

For segmental expansion exercise following procedure was followed: Placing therapist's hands along lateral aspect of lower ribs to fix the patient's attention to the areas at which movement is to occur and then patient was asked to breathe out, and feel the rib cage move downward and inward. As patient breathes out, firm pressure was placed into the ribs with palm of hand. Just prior to inspiration, application of a quick downward and inward stretch to the chest is done. Patient was told to expand the lower rib area against therapist's hands as he breathes in and application of gentle manual pressure to lower rib area as the patient breathes in and chest expands and ribs flares. As patient breathes out, assistance by gently squeezing the rib cage in a downward and inward direction was given. Segmental breathing exercises were given to the patient at three segments: Apical segment, Lateral basal segment and Posterior basal segment. ${ }^{11}$

In expiration through Red Rubber Balloon, patient was asked to inspire deeply through the nose and then exhale as much as he/she can in the Red Rubber Balloon.

Patients were given prone positioning and lateral recumbent positioning according to the need.

Active limb physiotherapy included of active movements of upper and lower limb.

Walking in ward and in ICU was made to perform by patient under supervision.

\section{STATISTICAL ANALYSIS:}

version 16.

Data was analysed using SPSS

Significance value was kept 0.05 . Non Parametric Wilcoxon signed rank test was applied.

\section{RESULTS}

All the 26 subjects included completed the study.

The overall mean and standard deviation for corona anxiety scale pre and post intervention was $2.84 \pm 1.86$ and $1.26 \pm 1.04$ respectively.

The overall mean and standard deviation for corona stress scales pre and post intervention was $46.19 \pm 1.62$ and $37.88 \pm 1.63$ respectively.

The overall mean and standard deviation for patient health questionnaire (phq-9) pre and post intervention was $3.92 \pm 2.41$ and $1.15 \pm 1.31$ respectively.

Significant improvement in the scores was noted. 
Isha Trivedi et.al. Effectiveness of physiotherapy on psychosocial factors in Covid-19 positive patients: an interventional study.

\section{CORONA ANXIETY SCALE}

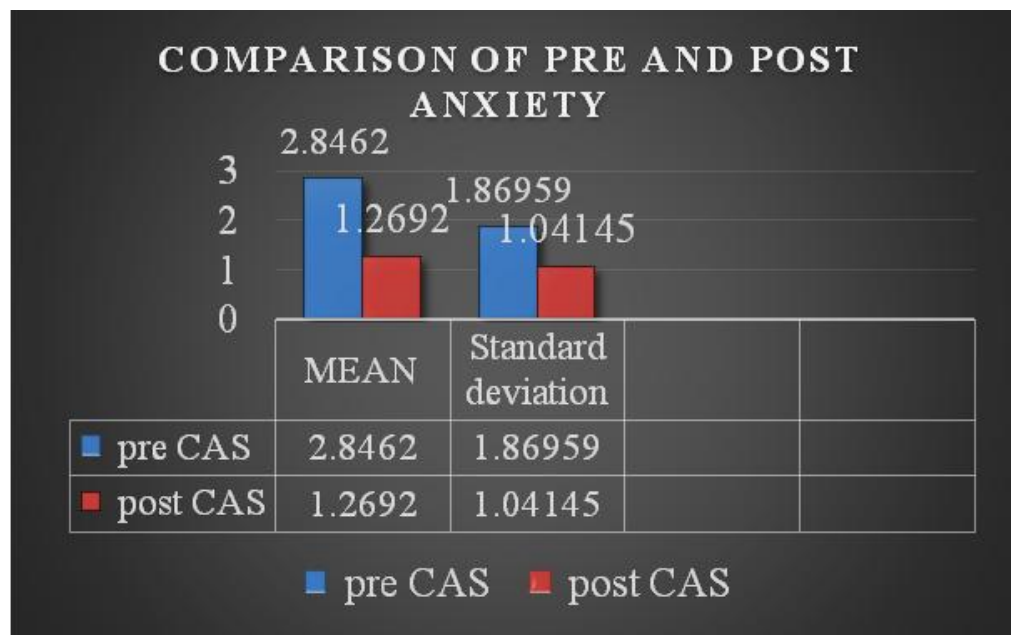

\section{CORONA STRESS SCALES}

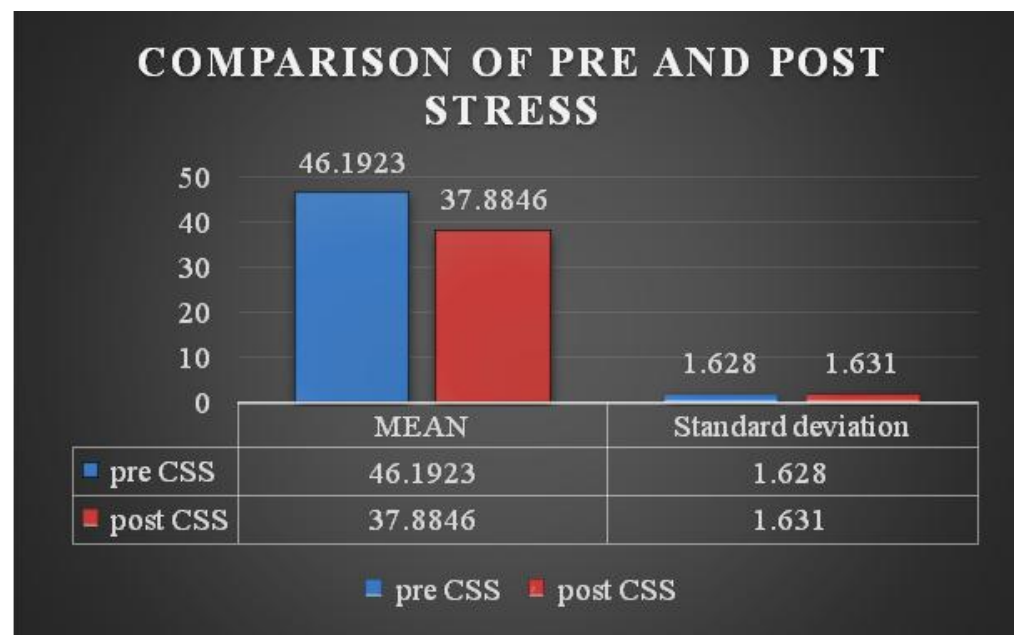

\section{PATIENT HEALTH QUESTIONNAIRE (PHQ-9)}

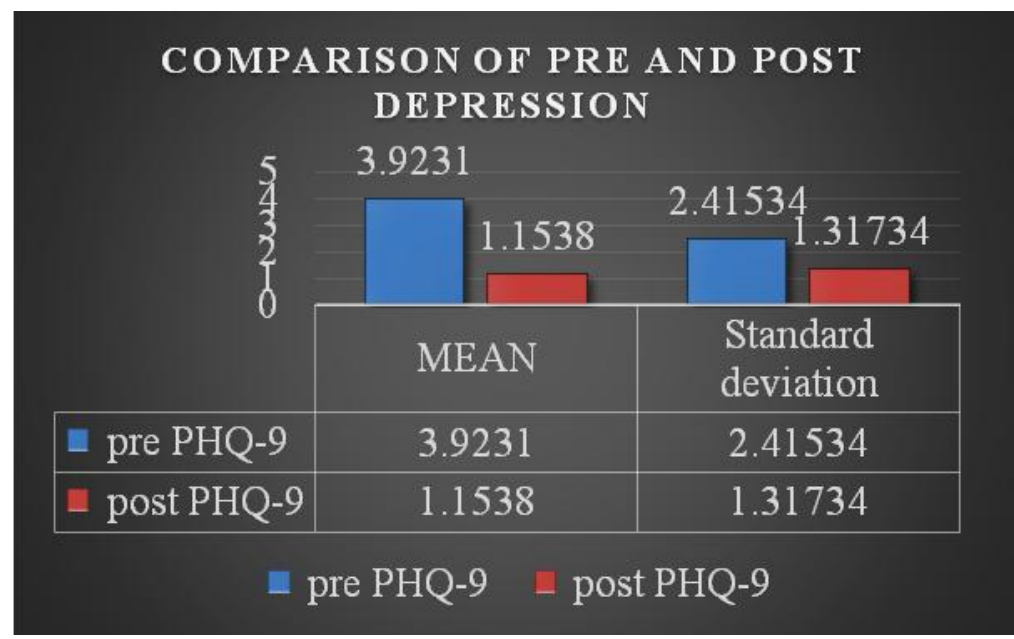

DISCUSSION

Covid19 positive patients suffer from various impairments which include respiratory, weakness and major psychosocial impact. Staying in isolated 
wards and ICUs, away from family also affects mental health.

The thoughts of post covid financial and social factors constantly hamper the patient.

According to the evidences, proper physiotherapy regimen if followed has a great impact in relieving adverse psychosocial factors.

A good mental support throughout the treatment is required. 26 patients were included in the interventional study. Prior assessment of stress, anxiety and depression was done.

Those patients were given the thorough physiotherapy regimen with proper mental support. After 14 days again the assessment was done. Significant improvement in the scores was noted.

Mental health of the hospitalized patients with COVID-19 during the epidemic remains unknown. Considering that patients after diagnosis of COVID-19 are more likely to have psychological concerns such as fear of progression of their illness, disability, or premature death. It is vital to investigate the prevalence and related factors of anxiety and depression in patients infected with COVID-19. ${ }^{12}$

Jiagi Xiong et al conducted a study on 'Impact of COVID-19 pandemic on mental health in the general population: A systematic review' and concluded that "The COVID-19 pandemic is associated with highly significant levels of psychological distress that, in many cases, would meet the threshold for clinical relevance. Mitigating the hazardous effects of COVID-19 on mental health is an international public health priority." 13

Those confirmed as having COVID19 may also undergo great psychological pressure and disturbance. ${ }^{14}$

Michael L. Tee et al conducted a study on 'Psychological impact of COVID-19 pandemic in the Philippines' and concluded that one-fourth of respondents reported moderate-to-severe anxiety and one-sixth reported moderate-to- severe depression and psychological impact.

\section{CONCLUSION}

Based on the results, physiotherapy proved to be effective in improving the psychosocial factors in the individuals who were covid19 positive.

The scores of anxiety, stress and depression significantly reduced after the intervention.

Hence it is proved that physiotherapy plays a positive role in management of psychosocial factors in covid19 patients and it should be included as an integral part of treatment protocol in managing the same.

\section{ACKNOWLEDGEMENTS}

Special thanks to my guide for always helping throughout the study. Grateful to the subjects who participated in my study. Thanks to my family and friends for always motivating me.

\section{Limitations of the Study:}

The control group was not taken

\section{Conflict of Interest: None}

\section{Source of Funding: None}

\section{Ethical Approval: Approved}

\section{REFERENCES}

1. Li H, Liu SM, Yu XH, Tang SL, Tang CK. Coronavirus disease 2019 (COVID-19): current status and future perspectives. International journal of antimicrobial agents. 2020 May 1;55(5):105951.

2. Batty GD, Deary IJ, Luciano M, Altschul DM, Kivimäki M, Gale CR. Psychosocial factors and hospitalisations for COVID-19: prospective cohort study based on a community sample. Brain, behavior, and immunity. 2020 Oct 1;89:569-78.

3. Lee SA. Coronavirus Anxiety Scale: A brief mental health screener for COVID-19 related anxiety. Death studies. $2020 \mathrm{Jul}$ 2;44(7):393-401.

4. Sotgiu G, Dobler CC. Social stigma in the time of coronavirus disease 2019. Eur 

interventional study.

Respir J. 2020 Aug; 56(2): 2002461. doi: 10.1183/13993003.02461-2020

5. Taylor S, Landry CA, Paluszek MM, Fergus TA, McKay D, Asmundson GJ. Development and initial validation of the COVID Stress Scales. Journal of Anxiety Disorders. 2020 May 1;72:102232.

6. Manea L, Gilbody S, McMillan D. A diagnostic meta-analysis of the Patient Health Questionnaire-9 (PHQ-9) algorithm scoring method as a screen for depression. General hospital psychiatry. 2015 Jan 1;37(1):67-75.

7. Richardson LP, McCauley E, Grossman DC, McCarty CA, Richards J, Russo JE, Rockhill C, Katon W. Evaluation of the Patient Health Questionnaire-9 Item for detecting major depression among adolescents. Pediatrics. 2010 Dec 1;126(6):1117-23 .

8. Westerdahl E. Optimal technique for deep breathing exercises after cardiac surgery. Minerva Anestesiol. 2015 Jun 1;81(6):67883.

9. Mendes LP, Moraes KS, Hoffman M, Vieira DS, Ribeiro-Samora GA, Lage SM, Britto RR, Parreira VF. Effects of diaphragmatic breathing with and without pursed-lips breathing in subjects with COPD. Respiratory care. 2019 Feb 1;64(2):136-44.

10. White D, Stiller K, Willson K. The role of thoracic expansion exercises during the active cycle of breathing techniques. Physiotherapy Theory and Practice. 1997 Jan 1;13(2):155-62.
11. Sarkar A, Sharma H, Razdan S, Kuhar S, Bansal N, Kaur G. Effect of segmental breathing exercises on chest expansion in empyema patients. Physiother Occup Ther. $2010 \mathrm{Jul} ; 4(3): 17$.

12. Kong $\mathrm{X}$, Zheng $\mathrm{K}$, Tang $\mathrm{M}$, Kong $\mathrm{F}$, Zhou J, Diao L, Wu S, Jiao P, Su T, Dong Y. Prevalence and factors associated with depression and anxiety of hospitalized patients with COVID-19. MedRxiv. 2020 Jan 1.

13. Xiong J, Lipsitz O, Nasri F, Lui LM, Gill H, Phan L, Chen-Li D, Iacobucci M, Ho R, Majeed A, McIntyre RS. Impact of COVID19 pandemic on mental health in the general population: A systematic review. Journal of affective disorders. 2020 Aug 8.

14. Khan KS, Mamun MA, Griffiths MD, Ullah I. The mental health impact of the COVID19 pandemic across different cohorts. International journal of mental health and addiction. $2020 \mathrm{Jul}$ 9:1-7.

15. Tee ML, Tee CA, Anlacan JP, Aligam KJ, Reyes PW, Kuruchittham V, Ho RC. Psychological impact of COVID-19 pandemic in the Philippines. Journal of affective disorders. 2020 Dec 1;277:379-91.

How to cite this article: Trivedi I, Shah S. Effectiveness of physiotherapy on psychosocial factors in COVID-19 positive patients: an interventional study. Int J Health Sci Res. 2021; 11(5): 323-328. DOI: https://doi.org/10.52403/ ijhsr.20210550 